\title{
Gender differences in COPD: authors' reply
}

\author{
Immacolata Ambrosino, ${ }^{1}$ Orazio Valerio Giannico, ${ }^{2}$ Cecilia Politi, ${ }^{3}$ Anna Maria Moretti ${ }^{4}$ \\ ${ }^{1}$ Local Healthcare Unit of Bari, Health District 13, Bari; ${ }^{2}$ Department of Biomedical Science, University of Bari Aldo Moro, \\ Bari; ${ }^{3}$ Department of Internal Medicine, Veneziale Hospital, Isernia; ${ }^{4}$ President of GISeG, Italian Group for Health and Gender, \\ Bari, Italy
}

Dear Editor,

we would like to clarify some points about Nicolini's et al. observations on the paper entitled Gender differences in COPD (chronic obstructive pulmonary disease): an analysis of hospitalization indicators. ${ }^{1}$

As described in the manuscript, our study was a retrospective observational descriptive analysis, performed in all the Apulian internal medicine wards from 2005 to 2016.

The gender differences in such a big sample of 93,638 discharged patients with a principal diagnosis of COPD with or without exacerbation $(74,007$ were acute exacerbation) were analyzed using the Hospital Discharge Register Database. In our study we considered the official nosological data, controlled by the Apulian regional government.

The ICD (International Statistical Classification) is used to code all diagnoses, symptoms and procedures recorded after hospital care. Furthermore, ICD database provides information not only about sex, but also on educational level, marital status, age, length of stay in hospital, re-hospitalization and comorbidities, hospital mortality. It is well known and pointed out in the discussion that the SDO (the Italian hospital discharge data source) does not provide any information about disease severity or smoking habits.

Is the DRG-based diagnosis the best way for a right analysis of gender differences in COPD? We do

Correspondence: Immacolata Ambrosino, Local Healthcare Unit of Bari, Health District 13, Corso Benedetto Croce 165, 70125 Bari, Italy.

E-mail: imma-ambrosino@libero.it

Received for publication: 4 March 2020.

Accepted for publication: 23 March 2020.

This work is licensed under a Creative Commons Attribution NonCommercial 4.0 License (CC BY-NC 4.0).

${ }^{\circ}$ Copyright: the Author(s), 2020

Licensee PAGEPress, Italy

Italian Journal of Medicine 2020; 14:112-113

doi:10.4081/itjm.2020.1273 not know. But we emphasize the fact that the DRG system and ICD data represent official data, published by our Health Ministry about the prevalence of COPD. Our national agency for regional health services (AGENAS) indicates a prevalence of COPD of $4.5 \%$ in Italy, according to anamnestic data and/or current presence of chronic bronchitis and emphysema, as approximate criteria, based on clinical practice in the real world. ${ }^{2}$ In addition, a recent study has validated the positive predictive value of ICD9-CM codes in the diagnosis of COPD. ${ }^{3}$

Acute, frail and complex elderly patients admitted to IM wards are not always able to collaborate in the execution of spirometric examinations. Moreover, spirometry can be indicated in DRG database but it does not modify the costs of hospitalization, so often it is not reported.

The addition of spirometry testing, as a prerequisite for diagnostic ICD9-CM codes for COPD, would obviously improve the diagnostic positive predictive value. ${ }^{3}$

In our study COPD patients were mostly elderly, frail and complex. We evaluated the differences in type and prevalence of comorbidities among male and female patients, considering the first 5 secondary diagnoses in the database. Our analysis revealed some important differences between the two sexes (in comorbidities and outcomes).

Heart failure and obesity are comorbidities considered in our paper. The supposed misdiagnosis between acute exacerbation of COPD, acute heart failure and obesity hypoventilation syndrome, is not the aim of our study. In addition, SDO data (administrative official data) do not allow to verify the accuracy of the main or of the secondary diagnosis.

Usually, patients with primary diagnosis of COPD exacerbation are admitted to the hospital after observation in the emergency room. Therefore, a COPD exacerbation which needs hospital admission is considered a letter C or D stage (GOLD 2019). In addition, the presence of active comorbidity is useful for assessing the severity of the COPD patient to secure the appropriate care setting.

The therapy code (ICD-9 93.9x) includes a lot of 
respiratory procedures. Their use in the coding of this acute COPD population can help to better define the severity of the illness.

In conclusion, results of our study are not to be underestimated in importance, as they are based on a large amount of real world data and could represent a fundamental tool for clinical decisions. Real world observational studies can be considered as an addition to RCTs and not as their substitutes, where EBM is applied and on which guidelines are based, filling important gaps in the scientific landscape.

With all the limits declared, our paper could be useful in the discussion on the gender differences in some important issues in COPD management. The increase in the hospitalization of COPD women, in the number of procedures, comorbidities and length of stay in hospital, have negative impact on the clinical progression as well as on the survival and quality of life. We need more opportunities to ensure better care, promoting pathways of assistance specifically tailored for male and female patients with COPD.

\section{References}

1. Ambrosino I, Gallone MS, Patano F, et al. Gender differences in chronic obstructive pulmonary disease: an analysis of hospitalization indicators. Ital $\mathrm{J} \mathrm{Med}$ 2019;13:38-44.

2. Rossi A, Butorac-Petanjek B, Chilosi M, et al. Chronic obstructive pulmonary disease with mild airflow limitation: current knowledge and proposal for future research - a consensus document from six scientific societies [published correction appears in Int J Chron Obstruct Pulmon Dis. 2019 Jan 18;14:247]. Int J Chron Obstruct Pulmon Dis 2017;12:2593-610.

3. Ho TW, Ruan SY, Huang CT, et al. Validity of ICD9-CM codes to diagnose chronic obstructive pulmonary disease from National Health Insurance claim data in Taiwan. Int J Chron Obstruct Pulmon Dis 2018;13:3055-63. 